\title{
Spectrum of One-Dimensional Anharmonic Oscillators
}

\author{
H. A. Alhendi ${ }^{1}$ and E. I. Lashin ${ }^{1,2}$ \\ ${ }^{1}$ Department of physics and Astronomy, College of Science, \\ King Saud University, Riyadh, Saudi Arabia \\ ${ }^{2}$ Department of Physics, Faculty of Science, \\ Ain Shams University, Cairo, Egypt
}

February 1, 2008

\begin{abstract}
We use a power-series expansion to calculate the eigenvalues of anharmonic oscillators bounded by two infinite walls. We show that for large finite values of the separation of the walls, the calculated eigenvalues are of the same high accuracy as the values recently obtained for the unbounded case by the inner-product quantization method. We also apply our method to the Morse potential. The eigenvalues obtained in this case are in excellent agreement with the exact values for the unbounded Morse potential.
\end{abstract}

PACS numbers: 03.65.Ge, 02.30.Hq

\section{Introduction}

The spectra of many important potential functions frequently encountered in quantum mechanics can not be obtained exactly. Moreover, in most cases, the conventional approximate methods commonly discussed in most standard textbooks(see, for example, [1]), while possibly leading to rough estimates, are either unsatisfactory or computationally complicated. A well-known important example in this respect is a sufficiently deep double-well potential, in which splitting of energy levels of two neighboring states, in particular the ground and first excited state, is extremely small because of the non-degenerate nature of energy eigenvalues of bound states for one-dimensional potentials $[1,2]$. Perturbation and semiclassical methods are of limited use in such a case, and the variational method, although providing upper bounds, requires carefully chosen trial functions. A need thus arises for a relatively simple and effective approximate method with a high degree of accuracy.

A variant of approximate methods and numerical techniques has recently been devised to calculate to high precision the spectrum of the one-dimensional symmetric anharmonic oscillators, with either a symmetric solution [3]-[7] or a symmetry-broken solution [8]-[10]. 
A method based on the Hill determinant has been developed in a recent study [8]. This method, known as the inner-product quantization procedure, uses the representation $\Psi(x)=\sum_{i} a_{i}(E) x^{i} R_{\beta}(x)$ of the wave function, where $R_{\beta}$ is an appropriate reference function. With a heuristic argument based on the Hill determinant method, it is shown that the zeros of the coefficient functions $a_{i}(E)$ approximate the exact bound state energies with increasing accuracy as $i \rightarrow \infty$. The method has been applied to several unbounded one-dimensional anharmonic oscillators, leading to highly accurate energy eigenvalues. In particular, for a sufficiently deep double-well, the splitting of the energies of the ground state energy and first excited state becomes apparent only after 26 significant digits.

It has already, however, been pointed out $[4,5]$ that not all eigenvalues obtained by the Hill determinant method should be allowed, since the boundary condition that $\Psi(x) \rightarrow 0$ as $|x| \rightarrow \infty$ is not incorporated into the method and may lead to incorrect results for some values of the coupling constants and for potentials in which $x=\infty$ is an irregular singular point of the Schrödinger equation. In addition, the choice of the arbitrary reference function is important, as emphasized in [8]: in general, it should fall off more slowly than the asymptotic form of the wave function.

The purpose of the present work is to apply the method of power-series expansion to some anharmonic potential functions bounded by two infinite walls. We show that the expansion of the wave function in the form $\Psi(x)=\sum_{i} a_{i} x^{i}$ in the finite interval $-L<x<L$ with infinite walls at $x= \pm L$ leads, even for moderate values of $L$, to the same high accuracy as has been achieved for the anharmonic potentials considered in [8]. The initial work in this direction was carried out by Barakat and Rosner [3], for the case of a pure quartic oscillator. These investigators showed that the lowerorder eigenvalues tend rapidly to the values of the unbounded oscillator as $L$ is made large. The method has subsequently been employed for the bounded pure anharmonic oscillators $x^{2 k}$, with $k=1, \cdots, 5$, and for shallow doubly anharmonic oscillators [4]. The power series in the finite range does not require introducing reference functions and accommodates all eigenvalues, since the boundary conditions are imposed at finite $x$. The problem concerning the convergence of the wave functions mentioned in [8] does not arise here.

The rest of the paper is organized as follows. In Sect. 2, we apply the method of power-series expansion to anharmonic potentials, specifically to a quartic, sextic, octic, dectic, duodectic and quartic double-well. Also in Sect. 2, we consider potential functions admitting power series-expansion, as exemplified by the Morse potential. In Sect. 3, we explain the method for obtaining the energy eigenvalues numerically, presenting our eigenvalue calculations and comparing them with the values obtained by the inner-product quantization method and with the exact values available for the Morse potential. In Sect. 4, we present our conclusions.

\section{Power-series expansion solution}

To calculate the eigenvalues and eigenfunctions of the anharmonic oscillators bounded by infinitely high potentials at $x= \pm L$, it is necessary to solve the eigenvalue equation (we here assume units $\hbar=1,2 m=1$ )

$$
\left[\frac{d^{2}}{d x^{2}}+E-V(x)\right] \Psi(x)=0
$$


with the boundary conditions $\Psi( \pm L)=0$. In the present work, we consider polynomial potential functions and potentials admitting power-series expansion. In the former case, our potentials $V(x)$ are of the form

$$
V(x)=\mu^{2} x^{2}+g x^{2 k}, \quad(k=2, \cdots, 6)
$$

Here the coupling constant $g>0$ and the mass parameter $\mu^{2}$ takes real values.

Using the power-series expansion

$$
\Psi=\sum_{l=0}^{\infty} a_{l} x^{l}
$$

in eq. (1), we obtain the following recurrence formula for the expansion coefficients:

$$
\begin{aligned}
a_{l}=\frac{g a_{l-2 k-2}+\mu^{2} a_{l-4}-E a_{l-2}}{l(l-1)}, & l \neq 0,1 \\
a_{l}=0, & l<0
\end{aligned}
$$

The symmetry of eq. (1) implies that the solutions fall into two classes, even and odd. Even solutions can be obtained by imposing $a_{0}=1, a_{1}=0$, and odd solutions can be obtained by imposing $a_{0}=0, a_{1}=1$.(We ignore normalization in both cases.) The energy eigenvalues $E$ are obtained from the condition $\Psi(L)=0$ for both even and odd solutions. Since we are dealing with potentials admitting power-series expansion for $|x|<L$, the power-series solutions of $\Psi(x)$ are convergent, by a well-known theorem in differential equations [11].

To illustrate the method for potential functions admitting power-series expansion, we choose the Morse potential. For this potential, which happens to be non symmetric, we can derive recurrence relations in a manner similar to the symmetric case(performing a power-series expansion for the potential and truncating it after certain power). The resulting power-series solution depends linearly on both $a_{0}$ and $a_{1}$ and in addition depends on $E$. Extracting the coefficient of $a_{0}$ and $a_{1}$ in the power-series solution and calling them $f^{0}$ and $f^{1}$, respectively, we then cast the wave function $\Psi(x, E)$ into a form suitable for applying the boundary conditions:

$$
\Psi(x, E)=f^{0}(x, E) a_{0}+f^{1}(x, E) a_{1} .
$$

The boundary conditions for $\Psi( \pm L, E)$ yield two homogeneous linear equations with $a_{0}$ and $a_{1}$ as unknowns:

$$
\begin{array}{r}
f^{0}(L, E) a_{0}+f^{1}(L, E) a_{1}=0 \\
f^{0}(-L, E) a_{0}+f^{1}(-L, E) a_{1}=0 .
\end{array}
$$

For there to exist a nontrivial solution for $\left\{a_{0}, a_{1}\right\}$, the following condition must be satisfied:

$$
f^{0}(L, E) f^{1}(-L, E)-f^{1}(L, E) f^{0}(-L, E)=0 .
$$


This fact allows us to solve for the unknown energies $E$ and the constants $a_{0}$ and $a_{1}$. For the case of the well-known Morse potential

$$
V(x)=V_{0}\left(1-e^{-\lambda x}\right)^{2},
$$

where $V_{0}$ and $\lambda$ are the depth and range parameters, respectively, the exact energy eigenvalues and eigenfunctions are given by

$$
\begin{aligned}
E_{n} & =2 \lambda \sqrt{V_{0}}\left[\left(n+\frac{1}{2}\right)-\left(n+\frac{1}{2}\right)^{2} \frac{\lambda}{2 \sqrt{V_{0}}}\right] \\
\Psi_{n}(x) & =N(n) y^{\alpha-n-\frac{1}{2}} e^{-y / 2} L_{n}^{2 \alpha-2 n-1}(y)
\end{aligned}
$$

where $n$ must be positive and less than $\frac{\sqrt{V_{0}}}{\lambda}-\frac{1}{2}$, and where $\alpha=\sqrt{\frac{V_{0}}{\lambda^{2}}} ; \quad y=2 \alpha e^{-\lambda x}$.

The functions $L_{n}^{a}(x)$ are the generalized Laguerre polynomials, while the normalization constant $N(n)$ is explicitly given by

$N(n)=\sqrt{\frac{\lambda(2 \alpha-2 n-1) n !}{\Gamma(2 \alpha-n)}}$.

To employ the method, we expand the potential in eq. 8 as power series, truncating the series beyond, say, $x^{30}$ :

$$
\begin{aligned}
V(x) \approx & V_{0}\left[x^{2} \lambda^{2}-x^{3} \lambda^{3}+\frac{7 x^{4} \lambda^{4}}{12}-\frac{x^{5} \lambda^{5}}{4}+\frac{31 x^{6} \lambda^{6}}{360}-\frac{x^{7} \lambda^{7}}{40}+\cdots\right. \\
& \left.+\frac{536870911 x^{30} \lambda^{30}}{132626429906095529318154240000000}\right] .
\end{aligned}
$$

\section{Calculation of Energy Eigenvalues}

To obtain the energy eigenvalues, we approximate the power series with a finite number of terms $\Psi_{n}(x, E)$. The boundary condition for a specific value of $L$ is $\Psi_{n}(L, E)=0$. To find the zeros of $\Psi_{n}(L, E)$ with respect to $E$, we first plot $\Psi_{n}(L, E)$ as a function of $E$. Around each zero, we locate two nearby points $E$ at which the function $\Psi_{n}(L, E)$ changes sign. We use these two points in the initial iteration of the "bisection method" for finding the zeros. In implementing the method, we use the Mathematica (version 3) package, relying extensively on its exact-number manipulation capabilities. We check the stability of the numerical results at a certain degree of accuracy for a particular L by increasing $n$ until the obtained value of $E$ stays fixed.

To illustrate the method, we present in Fig. 1 an example for the behaviour of even and odd solutions $\psi_{e}(E)$ and $\psi_{o}(E)$ for $L=2$ and $I=100$ (where $I$ indicates the number of nonvanishing terms in the truncated expansion of the wave function), for the case of the quartic anharmonic oscillator (potential $V(x)=x^{2}+x^{4}$ ).

We use the same procedure to obtain energies in the case of a non symmetric potential. In this case, however, we apply the condition given by eq. 7 . 
In Table 1, we present the calculated energies of the ground and first excited states for the bounded quartic anharmonic oscillator. Our method shows systematic convergence for increasing values of both $I$ and $L$, exceeding some of the high- accuracy results in[8]. As providing evidence for the accuracy attained, we present the ground-state energy with 300 digits in Table 1. We have checked the rate of convergence with a reference function of the type used in ref.[8]. Unfortunately, however, our study reveals that this reference-function procedure does not accelerate the rate of convergence, and that it makes the determination of the energy eigenvalues less reliable. Table 2 shows the calculated energies of the ground and first excited states for the bounded sextic, octic, dectic and doudectic anharmonic potentials, in a comparison with the values reported in [8] for the unbounded case.

Figure 1: The even solution $\psi_{e}(E)$ (broken curve) and odd solution $\psi_{o}(E)$ (solid curve) for the bounded quartic anharomnic oscillator (potential $V(x)=x^{2}+x^{4}$ ), where $2 L$ is the separation of the walls and $I$ is the number of non vanishing terms in the truncated expansion of the wave function.

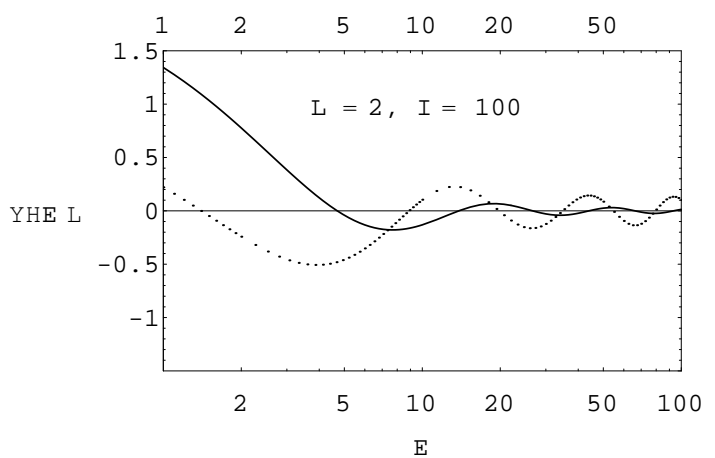

Figure 2 shows the dependence of the ground state energy for the bounded quartic anharmonic oscillator $\left(V(x)=x^{2}+g x^{4}\right)$ on the coupling constant $g$, for $0 \leq g \leq 10$, and $L=5$. It is clear that the ground-state energy increases, as expected, when the coupling constant is increased.

Figure 3 shows the wave functions for the ground state and first excited state in the case of the quartic anharmonic oscillator $\left(V(x)=x^{2}+g x^{4}\right)$ for $g=0,1,2$. Figure 4 shows the ground and first excited state for the sextic, octic, dectic and duodectic anharmonic potentials $V(x)=x^{2}+x^{2 k}$ (where $k=3,4,5,6$, respectively).

As emphasized in Sect. 2, all these graphs of the wave functions show the convergence of the power-series expansion in the finite interval.

Table 3 presents the calculated energies of the ground and first excited states for the bounded double-well potential, in comparison with the values reported in [8] for the unbounded case. Our calculations confirm the predictions made in [8] concerning the pseudo-degenerate nature of the ground-state and first-excited-state energies for $\mu^{2}=25$, which becomes apparent only after 26 significant digits. In addition, we present the energies of the ground state and first excited state for $\mu^{2}=35,50$. The splitting for $\mu^{2}=35$ and $\mu^{2}=50$ are revealed after 42 and 72 significant digits, respectively.

Our calculations confirm the general qualitative results that the energies of the lowlying states of the double-well potential become almost degenerate (pseudo-degenerate) as the depth of the well increases.

In the case of the Morse potential with the particular values $\lambda=1$ and $V_{0}=400$, 
Table 1: The calculated energies for the ground and first excited state for the bounded quartic anharmonic (potential $V(x)=x^{2}+x^{4}$ ), where $(2 L)$ is the width of the well and $I$ is the number of the nonvanishing terms in the truncated series of the wave function.

\begin{tabular}{|c|c|c|c|}
\hline$I$ & $L$ & $n$ & $E_{n}$ \\
\hline \multirow[t]{2}{*}{15} & 1 & 0 & 2.6365802 \\
\hline & & 1 & 10.2632444 \\
\hline \multirow[t]{2}{*}{25} & 2 & 0 & 1.39783341 \\
\hline & & 1 & 4.58734092 \\
\hline \multirow[t]{2}{*}{50} & 3 & 0 & 1.3923516 \\
\hline & & 1 & 4.648812 \\
\hline \multirow[t]{2}{*}{125} & 4 & 0 & 1.392351641530291855 \\
\hline & & 1 & 4.64881270421207753 \\
\hline \multirow[t]{2}{*}{250} & 5 & 0 & 1.392351641530291855657507876609934184 \\
\hline & & 1 & 4.6488127042120775363770329172605844 \\
\hline 2250 & 11 & 0 & 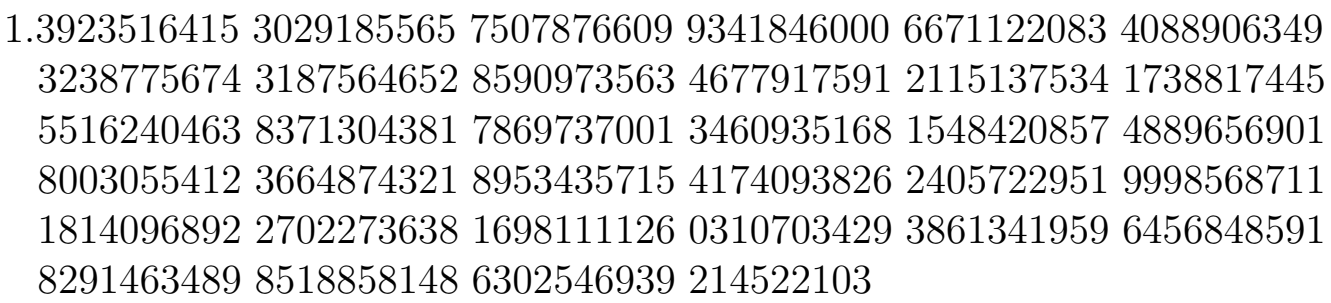 \\
\hline
\end{tabular}

Table 2: The calculated energies for the ground and first excited state for the bounded quartic, sextic, octic, dectic and duodectic anharmonic oscillators (with the indicated potentials $V(x)$ ), where $2 L$ is the width of the well, and where the underlined values are as calculated for the unbounded potential in [8].

\begin{tabular}{ccc}
\hline$V(x)$ & $n$ & $E_{n}$ \\
\hline$x^{2}+x^{6}$ & 0 & $\underline{1.43562461900339231576127222054252}$ \\
& 1 & 5.03339593772026647682838545349365 \\
& & $L=3$ \\
$x^{2}+x^{8}$ & 0 & $\underline{1.49101989566220496417108006064743}$ \\
& 1 & 5.36877806174812976635097601368018 \\
$x^{2}+x^{10}$ & 0 & $\underline{1.54626351257234572711783303167771}$ \\
& 1 & 5.65933772479004484069656154796369 \\
& & $L=2$ \\
$x^{2}+x^{12}$ & 0 & 1.59799049927600 \\
& 1 & 5.91264617503482 \\
\hline
\end{tabular}


Figure 2: Dependence of the ground state energy of the bounded quartic anharmonic oscillator (potential $\left.V(x)=x^{2}+g x^{4}\right)$ on the coupling constant.

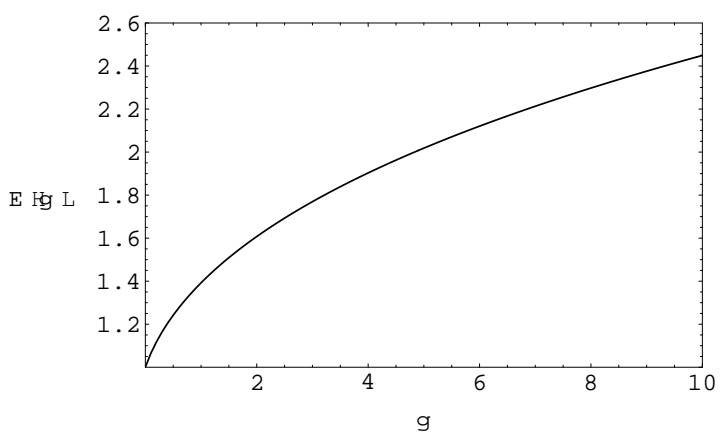

Figure 3: The wave functions for the ground (left) and first excited (right) for selected values of the coupling constant $g$, for the bounded quartic anharmonic oscillator (potential $V(x)=x^{2}+g x^{4}$ ).
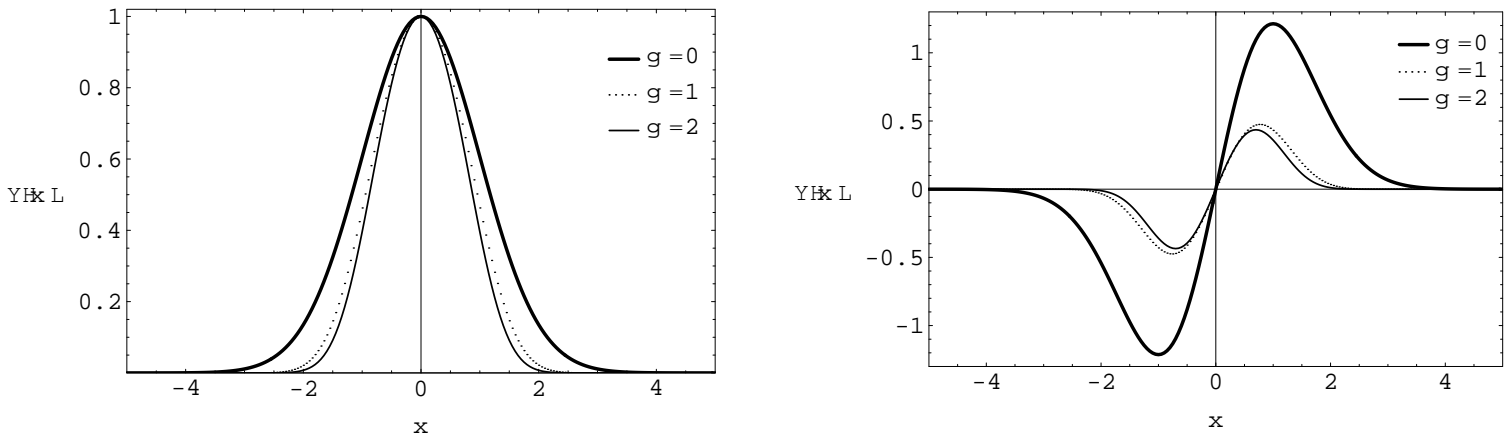

Figure 4: The solid curve represent the ground (left) and first excited (right) state wave function for the bounded sextic anharmonic oscillator $\left(V(x)=x^{2}+x^{6}\right)$. The dotted curves represent respectively (inward) the ground state wave functions for the bounded octic, dectic and duodectic case $(V(x)=$ $\left.x^{2}+x^{2 k}, k=4,5,6\right)$.
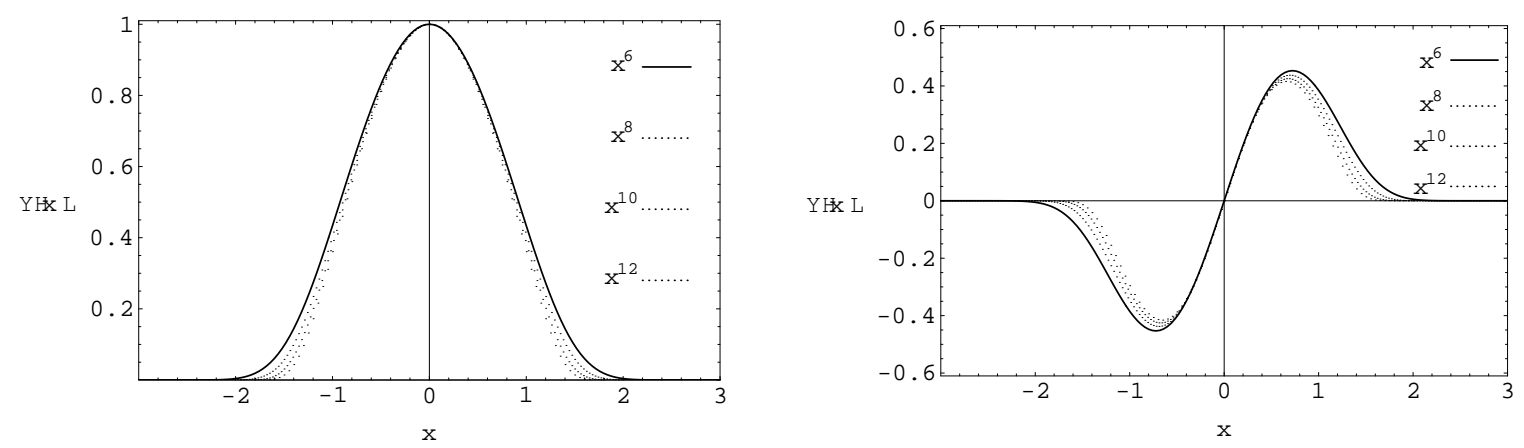
Table 3: The calculated energies for the ground and first excited state for the bounded potential $V(x)=-\mu^{2} x^{2}+x^{4}$, where $\mu$ is the mass parameter and $2 L$ is the width of the well, and where the underlined values are as calculated for the unbounded potential in [8].

\begin{tabular}{|c|c|c|}
\hline$\mu^{2}$ & Parity & $E_{ \pm}$ \\
\hline & & $L=6$ \\
\hline \multirow[t]{2}{*}{0} & + & 1.060362090484182899647046016692663545515208728529 \\
\hline & - & 3.7996730298013941687830941885125689577660065467327 \\
\hline \multirow[t]{2}{*}{1} & + & $\underline{0.657653005180715123059021723110593560374937941936}$ \\
\hline & - & $\underline{2.834536202119304214654676208748964958216940153453}$ \\
\hline \multirow[t]{3}{*}{5} & + & -3.41014276 1239829475297709653521909198712339047565 \\
\hline & - & -3.250675362289235980228513775547736877154601147639 \\
\hline & & $L=8$ \\
\hline \multirow[t]{2}{*}{10} & + & -20.63357670 2947799149958554837431508765315946057736 \\
\hline & - & -20.633546884404911079343874 100461390367842934101495 \\
\hline \multirow[t]{2}{*}{15} & + & -50.84138728 4381954366250996515741233774789627482985 \\
\hline & - & -50.841387284187005154710149735648634445905768683578 \\
\hline \multirow[t]{2}{*}{25} & + & -149.219456142190888029163966538165774475440692275913 \\
\hline & - & -149.219456142190888029163958974359019195734904923409 \\
\hline \multirow[t]{2}{*}{35} & + & -297.912194493707618450107115884174839453889352429871 \\
\hline & - & $\begin{array}{c}-297.912194493707618450107115884174839453889168865338 \\
L=9\end{array}$ \\
\hline \multirow[t]{2}{*}{50} & + & $\begin{array}{c}-615.020090902757816566217383210361567263581055195838 \\
06693413442605559721769729429892\end{array}$ \\
\hline & - & $\begin{array}{c}-615.020090902757816566217383210361567263581055195838 \\
06693413442605559721740320140809\end{array}$ \\
\hline
\end{tabular}


we obtain the energy eigenvalues tabulated in Table 4. Our values are identical with the exact values to the order indicated in the table. Figure. 5 shows the ground state and the first excited state, respectively, for a bounded Morse potential confined to the interval $-2 \leq x \leq 2$. These figures match the exact corresponding wave function of the unbounded Morse potential in the same interval.

Table 4: Energy eigenvalues for the Morse potential with depth parameter $V_{0}=400$ and range parameter $\lambda=1$, where the results for the bounded case are obtained for $I$ (the number of nonvanishing terms in the truncated series of the wave function) set to 500 and $L$ (the half-width of the well) set to 2 .

\begin{tabular}{ccccc}
\hline & $E_{0}$ & $E_{1}$ & $E_{2}$ & $E_{3}$ \\
\hline & 19.75000000000 & 57.75000000000 & 93.75000000000 & 127.75000000 \\
Exact & 19.75 & 57.75 & 93.75 & 127.75 \\
\hline
\end{tabular}

Figure 5: The wave function for ground (left) and first excited (right) state for the bounded Morse potential, with depth parameter $V_{0}=400$ and range parameter $\lambda=1$, where the results for the bounded case are obtained for $I$ (the number of nonvanishing terms in the truncated series of the wave function) set to 500 and $L$ (the half-width of the well) set to 2
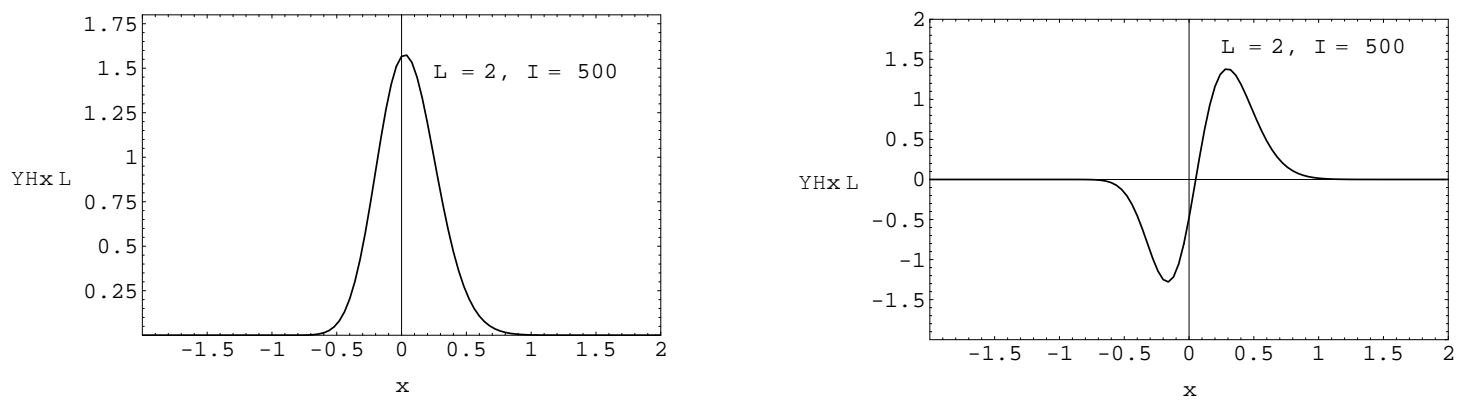

\section{Conclusion}

In this paper, we have applied the method of power-series expansion to a variety of symmetric one-dimensional anharmonic potential functions bounded by two infinite walls. We have compared our calculations of the low-lying energy levels with results from the inner-product quantization procedure. For all the potentials considered, we have chosen values for the number $n$ of terms in the power series and the distance $2 L$ between the walls that yield results of an accuracy superior to the results from the inner-product quantization procedure. More importantly, in analyzing the ground and first excited states of the deep double well, we have found the splitting of the energy levels to become apparent only after 26 significant digits for the mass parameter $\mu^{2}=25$, in agreement with the result obtained from the inner-product quantization procedure. We have extended our calculation to the case of $\mu^{2}=35$ and $\mu^{2}=50$, where the splitting shows up after 42 and 72 significant digits, respectively. For sextic, octic, dectic and duodectic oscillators, we have calculated the energy and wave function for the first excited states as well as for the 
ground states. Finally, we have applied our method to the well-known Morse potential, finding that our numerical results match the exact results.

A reason for the capability of the present method is that for a bound state, the wave function is spatially localized. This means that the probability density $|\Psi|^{2}$ has appreciable values in a finite region of space, outside which the probability density tends rapidly to zero. To obtain a good approximation, it is thus reasonable, as is shown by our numerical results for our chosen example of the Morse potential, to consider the corresponding problem in a finite interval, with a suitable width, bounded by two infinite walls.

Although the two methods are equally powerful for the potentials considered in the present work, the power-series expansion in the finite range is convergent for potentials admitting a power-series expansion in the same finite interval. The power-series method has been also applied to the case of one dimensional multi-well oscillator [12]. In addition, it has been used in [13] to justify numerical results based on the Zinn-Justin conjecture [14]. We show in work under preparation that the power-series method can be extended to the case of three-dimensional spherically symmetric potentials.

\section{Acknowledgement}

This work was supported by the Research Center, College of Science, King Saud University under project number Phys/1423/02.

\section{References}

[1] L. D. Landau and E. M. Lifshitz, Quantum mechanics (Pergamon Press, Oxford, 1977), 3rd ed.

[2] S. Coleman, In The Whys of Subnuclear Physics, edited by A. Zichichi (Plenum, New York, 1979), pp. 270-274.

[3] R. Barakat and R. Rosner, Phys. Lett. A 83, (1981) 149 .

[4] R. N. Chaudhuri and B. Mukherjee, J. Phys. A 16, (1983) 3193 ; ibid. 17, (1984) 3327 .

[5] A. Hautot, Phys. Rev. D 33, (1986) 437.

[6] A. Voros, J. Phys. A 27, (1994) 4653 ; ibid. 32, (1999) 5993.

[7] M. Trott quant-ph/0012147(2000).

[8] C. J. Tymczak, G. S. Japaridze, C. R. Handy, and X. -Q. Wang, Phys. Rev. Lett. 80, (1998) 3673 .

[9] I. V. Chebotarev, Ann. Phys. (NY) 273, (1999) 114.

[10] A. K. Roy, N. Gupta, and B. M. Deb, Phys. Rev. A 65, (2002) 012109.

[11] E. A. Coddington, An introduction to ordinary differential equations, (Prentice-Hall,Inc., Englewood Cliffs, N.J., 1966), pp. 138-142. 
[12] H. A. Alhendi and E. I. Lashin quant-ph/0306016 (2003).

[13] H. A. Alhendi and E. I. Lashin, J. Phys. A 38, (2005) 6785 , quant-ph/0402101 (2004).

[14] U. D. Jentschura and J. Zinn-Justin, J. Phys. A 34, (2001) L253 . 